\title{
Physical activity in asthma control and its immune modulatory effect in asthmatic preschoolers
}

\author{
Debbie J. Maurer ${ }^{1,2,3}$ (1) | Chengyao Liu' ${ }^{1,4}$ ( \\ Claus Bachert $^{6}$ (1) | Susetta Finotto ${ }^{7}$ (1) | Ya-Dong Gao ${ }^{8}$ (i) | Anna Graser ${ }^{7}$ |

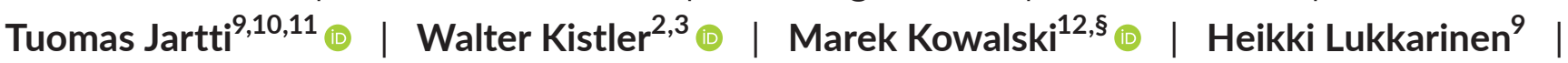 \\ Maria Pasioti ${ }^{5}$ | Ge Tan ${ }^{1}$ (1) | Michael Villiger ${ }^{2,3}$ | Luo Zhang,13 | Nan Zhang ${ }^{6}$ |

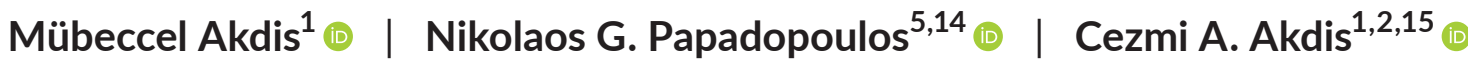

${ }^{1}$ Swiss Institute of Allergy and Asthma Research (SIAF), University of Zurich, Davos, Switzerland

${ }^{2}$ Swiss Research Institute for Sports Medicine (SRISM), Davos, Switzerland

${ }^{3}$ Department of Sports Medicine, Davos Hospital, Davos, Switzerland

${ }^{4}$ Department of Otolaryngology Head and Neck Surgery, Beijing TongRen Hospital, Capital Medical University, Beijing, China

${ }^{5}$ Allergy Department, 2nd Pediatric Clinic, National and Kapodistrian University of Athens, Athens, Greece

${ }^{6}$ Upper Airway Research Laboratory, Ghent University Hospital, Ghent, Belgium

${ }^{7}$ Department of Molecular Pneumology, Friedrich-Alexander-Universität (FAU) Erlangen-Nürnberg, Universitätsklinikum Erlangen, Erlangen, Germany

${ }^{8}$ Department of Allergology, Zhongnan Hospital of Wuhan University, Wuhan, China

${ }^{9}$ Department of Pediatrics and Adolescent Medicine, University of Turku and Turku University Hospital, Turku, Finland

${ }^{10}$ PEDEGO Research Unit, Medical Research Center, University of Oulu, Oulu, Finland

${ }^{11}$ Department of Pediatrics and Adolescent Medicine, Oulu University Hospital, Oulu, Finland

${ }^{12}$ Department of Immunology, Rheumatology and Allergy, Central University Hospital, Lodz, Poland

${ }^{13}$ Department of Allergy, Beijing TongRen Hospital, Capital Medical University, Beijing, China

${ }^{14}$ Division of Infection, Immunity \& Respiratory Medicine, University of Manchester, Manchester, UK

${ }^{15}$ Christine Kühne-Center for Allergy Research and Education, Davos, Switzerland

\section{Correspondence}

Cezmi A. Akdis, Swiss Institute of Allergy and Asthma Research (SIAF), University of Zurich, Herman-Burchard-Strasse 9, 7265 Davos, Wolfgang, Switzerland.

Email: akdisac@siaf.uzh.ch

\begin{abstract}
Background: The impact of physical activity on immune response is a hot topic in exercise immunology, but studies involving asthmatic children are scarce. Our aims were to examine whether there were any differences in the level of physical activity and daily TV attendance, to assess its role on asthma control and immune responses to various immune stimulants.
\end{abstract}

\begin{abstract}
Abbreviations: AIRE, Asthma Insights and Reality in Europe (study); BMI, Body Mass Index; CA, State of California; CCL, chemokine (C-C motif) ligand; CXCL, chemokine (C-X-C motif) ligand; EIA, exercise-induced asthma; FBS, fetal bovine serum; GINA, Global Initiative for Asthma; HEPES, N-2-hydroxyethylpiperazine-N'-2-ethanesulfonic acid; ICS, inhaled corticosteroids; IFN, interferon; IL, interleukin; MEM, Minimum Essential Medium; MET, metabolic equivalent; MyD88, myeloid differentiation primary response protein; NF-kB, nuclear factor kappa-light-chain-enhancer of activated B cells; PA, physical activity; PBMC, peripheral blood mononuclear cell; PBS, phosphate buffered saline; PEF, peak expiratory flow; PHA, phytohemagglutinin; Poly I:C, polyinosinic:polycytidylic acid; PreDicta, Post-infectious immune reprogramming and its association with persistence and chronicity of respiratory allergic diseases (study); R848, Resiquimod; RNA, ribonucleic acid; RPMI, Roswell Park Memorial Institute Medium; SD, standard deviation; TLR, Toll-like receptor; TNF- $\alpha$, tumor necrosis factor alpha; TV, television; TVA, television attendance; WHO, World Health Organization; Zymo, zymosan.
\end{abstract}

Debbie J. Maurer and Chengyao Liu equal first-author contribution.

Mübeccel Akdis, Nikolaos G. Papadopoulos and Cezmi A. Akdis are shared last authors.

${ }^{\S}$ Deceased.

This is an open access article under the terms of the Creative Commons Attribution-NonCommercial-NoDerivs License, which permits use and distribution in any medium, provided the original work is properly cited, the use is non-commercial and no modifications or adaptations are made.

(c) 2021 The Authors. Allergy published by European Academy of Allergy and Clinical Immunology and John Wiley \& Sons Ltd. 
Funding information

PreDicta; EU 7th framework programme, Grant/Award Number: HEALTH-F22010-260895; Swiss National Science Foundation
Methods: Weekly physical activity and daily television attendance were obtained from questionnaires at inclusion of the PreDicta study. PBMC cultures were stimulated with phytohemagglutinin (PHA), R848, poly I:C, and zymosan. A panel of cytokines was measured and quantified in cell culture supernatants using luminometric multiplex immunofluorescence beads-based assay.

Results: Asthmatic preschoolers showed significantly more TV attendance than their healthy peers ( $58.6 \%$ vs. $41.5 \% 1-3$ h daily and only $25.7 \%$ vs. $47.2 \% \leq 1$ h daily) and poor asthma control was associated with less frequent physical activity (PA) (75\% no or occasional activity in uncontrolled vs. $20 \%$ in controlled asthma; $25 \% \geq 3$ times weekly vs. 62\%). Asthmatics with increased PA exhibited elevated cytokine levels in response to polyclonal stimulants, suggesting a readiness of circulating immune cells for type 1, 2, and 17 cytokine release compared to subjects with low PA and high TV attendance. This may also represent a proinflammatory state in high PA asthmatic children. Low physical activity and high TV attendance were associated with a decrease in proinflammatory cytokines. Proinflammatory cytokines were correlating with each other in in vitro immune responses of asthmatic children, but not healthy controls, this correlation was more pronounced in children with sedentary behavior. Conclusion: Asthmatic children show more sedentary behavior than healthy subjects, while poor asthma control is associated with a substantial decrease in physical activity. Our results suggest that asthmatic children may profit from regular exercise, as elevated cytokine levels in stimulated conditions indicate an immune system prepared for responding strongly in case of different types of infections. However, it has to be considered that a hyperinflammatory state in high PA may not be beneficial in asthmatic children.

\section{KEYWORDS}

asthma, cytokines, immune modulation, physical activity, PreDicta

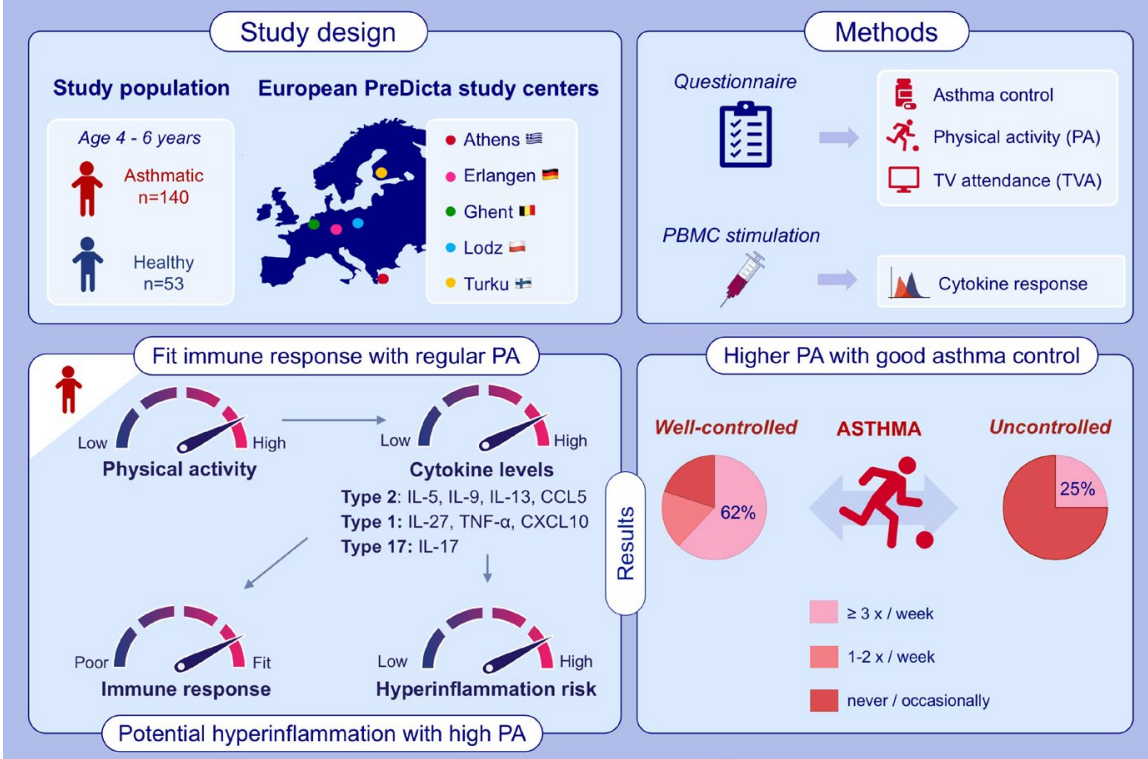

\section{GRAPHICAL ABSTRACT}

Asthmatic children show more sedentary behavior than healthy peers, especially those with uncontrolled asthma. Regular moderate-tohigh PA is associated with elevated cytokine levels in response to viral, bacterial, and fungal stimulations in asthmatics, which indicates a strong immune response and readiness to respond upon infections. High cytokine levels associated with high PA might also indicate hyperinflammation, suggesting the need for individual assessment to find an optimum PA level. 


\section{1 | INTRODUCTION}

Asthma affects more than 300 million people worldwide, and it is estimated that this number will increase to over one billion by 2050 . $^{1}$ It is more prevalent in children, where it lies among the top 20 chronic diseases for the global ranking of disability-adjusted life years ${ }^{1,2}$ with up to $25 \%$ of children affected in Western urban areas. ${ }^{3}$ The global burden of asthma in children and adolescents increased significantly over the last decades, while previously countries affected at lesser extent in Africa, South America, and Asia started to catch up on these numbers. ${ }^{1,4}$ Multiple factors are thought to play a role in this worldwide increase in asthma prevalence including allergen exposure and sensitization, environmental influences, viral infections, urbanization, diet, and sedentary lifestyle with physical inactivity. ${ }^{5,6}$ Asthma attacks often appear during physical activity (PA), ${ }^{7}$ termed "exerciseinduced asthma" (EIA) affecting $70-90 \%$ of asthmatic children. ${ }^{3}$

PA is beneficial both for growth and the psychological development of children. ${ }^{8-10}$ Although the World Health Organization (WHO) recommends for children and adolescents aged 5-17 years to be moderately to vigorously active for a minimum of $60 \mathrm{~min}$ daily, ${ }^{10,11}$ specific guidelines for children suffering from asthma are lacking. ${ }^{12}$ Although it is agreed that it is essential for asthmatic children to participate in sports, ${ }^{7}$ fear of EIA might prevent the practice of regular PA in children, particularly the ones with severe and/or uncontrolled asthma. ${ }^{3,13}$ This self-limiting cycle of inactivity has led to the common perception that asthmatics are more physically inactive in comparison with healthy individuals. ${ }^{3}$

Asthma pathogenesis is a consequence of immune dysregulation and epithelial barrier dysfunction, ${ }^{14}$ and research has shown that there are several underlying pathophysiological mechanisms (endotypes) that can lead to variable clinical presentations (phenotypes). ${ }^{15-17}$ An imbalance of both Th1/Th2 cells and Th17/Treg cells can play a crucial role in its development. ${ }^{18-20}$ Type 2 inflammation is considered to be the major driver in the most common phenotype, allergic asthma, ${ }^{21}$ and seems to be the result of a complex cross-talk between airway epithelium, innate and adaptive immunity. ${ }^{19}$ PA is known to impact both the innate and adaptive immune responses in various ways: For example, moderate exercise was found to lead to an acute increase in the absolute numbers of natural killer (NK) cells and NK cell cytotoxicity. ${ }^{22,23}$ As for the adaptive immune response, increased and intensified training loads were shown to lead to a reduction of T-cell functionality in well-trained individuals, with lower numbers of circulating type $1 \mathrm{~T}$ cells, reduced T-cell proliferation responses, and B-cell immunoglobulin synthesis. ${ }^{22}$ While Walsh et al. describe in their position statement on immune function and exercise a resulting temporary inhibition of Th1 cytokine production in trained individuals without asthma, studies examining the impact of aerobic exercise in murine asthma models reported an enhancement of Th1 and Treg responses and lower levels of Th2 cytokines. ${ }^{22,24,25}$

The "Post-infectious immune reprogramming and its association with persistence and chronicity of respiratory allergic diseases" (PreDicta) study was designed to prospectively evaluate asthma persistence in preschoolers in association with microbial exposures and immunological responses. ${ }^{26}$ PreDicta has demonstrated differential immune responses to viruses in asthma, ${ }^{27,28}$ as well as evolution of airway inflammation at that age. ${ }^{29}$ The objective of the present study was as follows: (a) to evaluate whether there were any differences in the level of PA and sedentary behavior between asthmatic and non-asthmatic children, (b) to assess the impact of asthma control on PA, and (c) to examine the influence of PA and long-time TV attendance on the immune system in asthmatic children. We have investigated multiple cytokine levels in response to four different immune stimuli: by phytohemagglutinin (PHA) that mimics polyclonal innate immune activation, by polyinosinic-polycytidylic acid (poly I:C) and R848 (resiquimod) that both mimic respiratory virus stimulation and by zymosan (zymo) that mimics fungal stimulation in PBMC cultures. R848 is an imidazoquinoline compound with potent antiviral activity that functions through the Toll-like receptor (TLR)7/TLR8 myeloid differentiation primary response protein (MyD88)-dependent signaling pathway. ${ }^{30}$ Poly I:C is a synthetic analog of double-stranded RNA that induces the production of proinflammatory cytokines by the activation of NF-kB through TLR3. ${ }^{31}$ Zymosan is a glucan binding to TLR2 and dectin1 found on the surface of yeasts. ${ }^{32}$

\section{2 | METHODS}

\section{1 | Study population \& design}

Our analyses were carried out within the framework of the PreDicta study, a 2-year multi-center prospective cohort study that has been conducted across five major European cultural and climatic regions (Greece, Germany, Belgium, Poland, and Finland). ${ }^{26}$

We included 140 pre-school children aged 4-6 years with a diagnosis of mild-to-moderate asthma confirmed by a doctor (in the preceding 2 years, according to the GINA guidelines 2005) as cases, which were previously recruited for the PreDicta study. Those children were all out-patients in their local hospital's asthma clinic and were included in the PreDicta study upon (parental) informed consent and fulfillment of eligibility criteria. Eligibility criteria for the PreDicta study included the ability to perform a peak expiratory flow (PEF) measurement at least once and a minimum of 3 wheezing episodes within the last 12 months prior to study inclusion (one of them within the last six months). Exclusion criteria were severe asthma, more than six courses of oral steroids during the last 12 months prior to study inclusion, immunotherapy, chronic medication use, or the history of chronic respiratory disease other than asthma and/ or allergic rhinitis (eg, cystic fibrosis). Additional exclusion criteria were an asthma exacerbation and/or upper respiratory tract infection within 4 weeks prior to study inclusion. The cross-sectional control group was recruited from surgical wards undergoing a planned minor operation (blood sampling was already scheduled as part of the standard diagnostic procedures). The control group consisted of 53 healthy, age- and center-matched children with no history of asthma/wheezing or any other allergic disease. Recruitment of both cohorts took place at the same time. 
WILEY-Allergy

\begin{tabular}{|c|c|c|c|}
\hline Patient characteristics & $\begin{array}{l}\text { Healthy controls } \\
(n=53)\end{array}$ & $\begin{array}{l}\text { Asthmatic patients } \\
(n=140)\end{array}$ & $p$ value \\
\hline Gender (F/M) & $31 / 22$ & $58 / 82$ & 0.04 \\
\hline Age $(y)$ mean $\pm S D$ & $5.0 \pm 0.8$ & $5.3 \pm 0.7$ & ns \\
\hline Wheezer (yes), n (\%) & $0(0 \%)$ & $140(100 \%)$ & $<.0001$ \\
\hline \multicolumn{4}{|l|}{ Atopic diseases (yes), $n$ (\%) } \\
\hline Allergic rhinitis, $n$ (\%) & $0(0 \%)$ & 89 (64\%) & $<.0001$ \\
\hline Atopic dermatitis, $n(\%)$ & $0(0 \%)$ & $73(52 \%)$ & $<.0001$ \\
\hline Adverse food reaction, $n(\%)$ & $0(0 \%)$ & $44(31 \%)$ & $<.0001$ \\
\hline \multicolumn{4}{|l|}{ Asthma control, $n(\%)$} \\
\hline Controlled & - & $74(53 \%)$ & - \\
\hline Partly controlled & - & $50(36 \%)$ & - \\
\hline Uncontrolled & - & $16(11 \%)$ & - \\
\hline \multicolumn{4}{|c|}{ Vigorous physical activity (times per week), $n(\%)$} \\
\hline Never or occasionally & $13(25 \%)$ & $42(30 \%)$ & ns \\
\hline Once/twice & $10(19 \%)$ & $24(17 \%)$ & ns \\
\hline Three times or more & $30(57 \%)$ & $75(53 \%)$ & ns \\
\hline \multicolumn{4}{|c|}{ TV attendance (hours per day), $n$ (\%) } \\
\hline$\leq 1 \mathrm{~h}$ & $25(47 \%)$ & $36(26 \%)$ & $<.05$ \\
\hline $1-3 h$ & $22(42 \%)$ & $82(59 \%)$ & $<.05$ \\
\hline$\geq 3 \mathrm{~h}$ & $6(11 \%)$ & $22(16 \%)$ & ns \\
\hline
\end{tabular}

TABLE 1 Patient characteristics, allergic comorbidities, asthma control, physical activity, and TV attendance in asthmatic and healthy preschoolers

Statistically significant values are shown bold.

\begin{tabular}{|c|c|c|c|c|}
\hline Asthma control & $\begin{array}{l}\text { Controlled } \\
(n=74)\end{array}$ & $\begin{array}{l}\text { Partially controlled } \\
(n=50)\end{array}$ & $\begin{array}{l}\text { Uncontrolled } \\
(n=16)\end{array}$ & $p$ value \\
\hline \multicolumn{5}{|c|}{ Vigorous physical activity (times per week), $n$ (\%) } \\
\hline Never or occasionally & $15(20 \%)$ & $14(28 \%)$ & $12(75 \%)$ & $<.0001$ \\
\hline Once/twice & $13(18 \%)$ & $11(22 \%)$ & $0(0 \%)$ & ns \\
\hline Three times or more & $46(62 \%)$ & $25(50 \%)$ & $4(25 \%)$ & $<.05$ \\
\hline \multicolumn{5}{|c|}{ TV attendance (hours per day), $n$ (\%) } \\
\hline$\leq 1 \mathrm{~h}$ & $22(30 \%)$ & $11(22 \%)$ & $3(19 \%)$ & ns \\
\hline$>1 \mathrm{~h}$ & $52(70 \%)$ & 39 (78\%) & $13(81 \%)$ & ns \\
\hline
\end{tabular}

TABLE 2 Asthma control, weekly physical activity, and daily TV attendance in asthmatic preschoolers

Statistically significant values are shown bold.

At recruitment, a detailed questionnaire on demographic characteristics, as well as asthma activity and control was handed out. Asthma was classified as uncontrolled, partly controlled, or controlled according to the GINA guidelines. ${ }^{12}$ As part of the questionnaires, weekly vigorous PA and daily television attendance (TVA) were assessed. PA with an intensity higher than 4 metabolic equivalents (METs) was classified as vigorous and included brisk walking, bicycling, gymnastics, dancing, basketball, soccer, athletics, tennis, swimming, jumping rope, and general participation in active outdoors games. Such activity had to be continuous and cause sweating and heavy breathing for periods longer than $15 \mathrm{~min}$.

Whole blood was obtained at baseline for the analysis of cytokine responses of peripheral blood mononuclear cells (PBMCs) cultured in the presence of various stimuli.

Written informed consent was obtained from parents/guardians of all the children recruited in the PreDicta study. The study protocol was approved by the local Ethics Committee of all participating institutions.

\section{2 | Blinding of study subjects}

The study participants were assigned individual study numbers, in which the first digit stands for the respective study center (1: Athens, 2: Erlangen, 3: Ghent, 4: Lodz, 5: Turku). The participants' full name could only be accessed by the clinical investigator and study nurses of the respective Childrens' Hospital.

\section{3 | In vitro culture of peripheral blood mononuclear cells}

Blood samples were collected in tubes with lithium heparin (Vacutainer ${ }^{\circledR}$ ) and diluted with an equal volume of warm PBS (Gibco, Invitrogen, Massachusetts). PBMCs were isolated by centrifuging at $800 \mathrm{~g}$ for $20 \mathrm{~min}$ at $18-20^{\circ} \mathrm{C}$ on Biocoll separating solution (Biochrom AG, Germany). PBMCs were washed three times, and the cell pellet was resuspended 
in complete medium [RPMI 1640 with HEPES $25 \mathrm{mM}$ and L-Glutamine (Gibco, Life Technologies Ltd, UK), supplemented with $10 \mathrm{ml} / \mathrm{L}$ PenicillinStreptomycin USA, $50 \mu \mathrm{l} / \mathrm{L} 1 \mathrm{M} \beta$-mercaptoethanol, $20 \mathrm{ml} / \mathrm{L}$ L-Glutamine plus MEM Vitamin, 20 ml/L Non-essential Amino Acid, Sodium Pyruvate and $10 \%$ heat-inactivated FBS (all from Sigma-Aldrich, Germany)]. The suspension was seeded in a flat-bottom 48-well tissue plate (Corning Incorporated, Costar, New York), with $5 \times 10^{5}$ viable cells per well ( $500 \mu \mathrm{ll}$ ). PBMCs were cultured in duplicates either with complete medium alone (unstimulated control) or with one of the following stimulants: $4 \mu \mathrm{g} / \mathrm{ml}$ Resiquimod (R848), $10 \mu \mathrm{g} / \mathrm{ml}$ Zymosan (InvivoGen, France), $10 \mu \mathrm{g} / \mathrm{ml}$ Phytohemagglutinin (PHA-M), $20 \mu \mathrm{g} / \mathrm{ml}$ Polyinosinic-polycytidylic acid potassium salt (Poly I:C), (Sigma-Aldrich, Germany), at $37^{\circ} \mathrm{C}, 5 \% \mathrm{CO}_{2}$. Cultures were harvested after $48 \mathrm{~h}$ and, after centrifugation at $600 \mathrm{~g}$ for $5 \mathrm{~min}$, supernatants were stored at $-80^{\circ} \mathrm{C}$ until analysis.

\section{4 | Detection of cytokines secreted in PBMCs culture supernatants}

Cytokine expression levels in the supernatants of stimulated cultures were quantified by multiplex bead-based fluorometric immunoassay (Milliplex, Millipore) using Luminex xMAP technology (Luminex 200, Bio-Rad) at the Swiss Institute for Allergy and Asthma Research (SIAF) in Davos, Switzerland. Values out of range were either marked as the lowest (those on a lower end) or the highest (those on a higher end) detectable values. The panel used contained IFN $\alpha 2$, IFN $\gamma$, IFN $\lambda$-2, IL1 $\beta$, IL-5, IL-6, IL-7, IL-9, IL-10, IL-12p70, IL-13, IL-17A, IL-23A, IL-25, IL-27, IL-33, CCL3, CCL4, CCL5, CXCL8, CXCL10, and TNF- $\alpha$. The T-cell subset classification of all measured cytokines can be found in Table S1.

\section{5 | Statistical analysis}

Nominal variables are presented as absolute and relative (\%) frequencies. Continuous variables are presented in violin plots to show frequency distribution, with median and quartiles. Data were evaluated for significant statistical differences using (a) the Chi-square test to evaluate associations between two nominal variables, (b) the non-parametric Kruskal-Wallis test for multiple comparisons, and (c) the non-parametric Mann-Whitney test for independent sample tests (Prism 8.0 for macOS; GraphPad Software, San Diego, CA, USA). Correlation was assessed with the Spearman method. A local regression fitting line was added to the scatter plot with a confidence level of $95 \%$. Statistical significance was set at $p<.05\left({ }^{*} p<.05 ;{ }^{* *} p<.01 ;{ }^{* * *} p<.001 ;{ }^{* * * *} p<.0001\right)$.

\section{3 | RESULTS}

\section{1 | Baseline characteristics}

From 167 cases and 66 controls that were recruited for the PreDicta cohort, a total of 140 asthmatic children (82 males, 59\%) and 53 healthy controls ( 22 males, $42 \%$ ) were included in this study. The reason for the exclusion of 27 subjects was insufficient blood samples for cell culture experiments. The respective number of cases and controls per study center is as follows: Athens $36 / 13$, Erlangen 22/15, Ghent 20/13, Lodz 25/4, and Turku 37/8. Mean age at recruitment was $5.3 \pm 0.7$ in asthmatic and $5.0 \pm 0.8$ in healthy children. The majority of asthma cases additionally suffered from allergic rhinitis (64\%) and more than half of them (52\%) reported atopic dermatitis, while $31 \%$ had experienced adverse food reactions.

Table 1 shows demographic characteristics, weekly vigorous PA and daily TVA as well as asthma control and allergic comorbidities, as gathered through questionnaires at inclusion. There were more boys in the asthmatic cohort (59\% versus $42 \%$ in the healthy cohort).

\subsection{Physical activity and TV attendance in asthmatic preschoolers and the effect of asthma control}

In the asthmatic group, a significantly higher daily TVA was reported (daily TVA $<1 \mathrm{~h}$ in $26 \%$ of cases versus $47 \%$ of controls; $1-3 \mathrm{~h}$ daily TVA in $59 \%$ of cases and $42 \%$ of controls, Table 1). Also, there was a higher percentage of children that were never or only occasionally

\section{(A) \\ Physical activity}

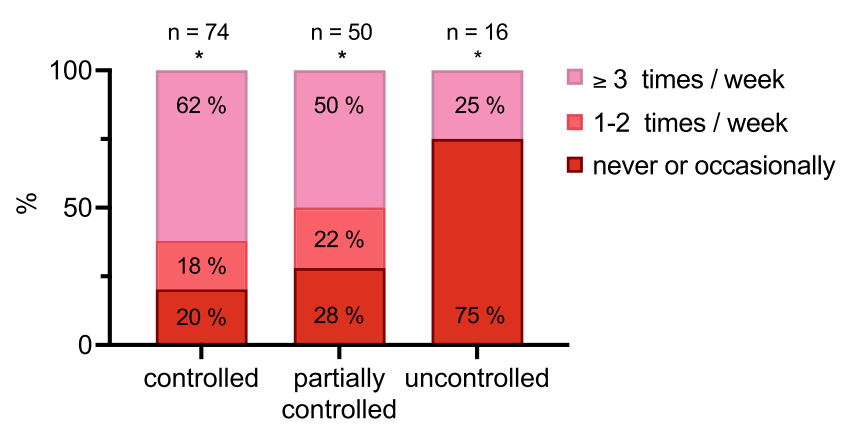

(B)

TV attendance

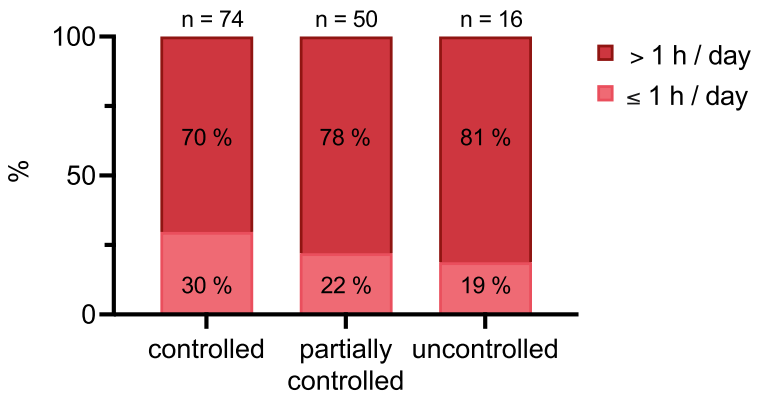

FIGURE 1 Impact of asthma control on weekly physical activity (A) and TV attendance (B). (A) Fraction of total (\%) analysis of subjects with vigorous $\mathrm{PA} \geq 3$ times a week, 1-2 times a week or never/occasionally physically active, grouped according to asthma control. (B) Fraction of total (\%) analysis of subjects with daily TV attendance $>1 \mathrm{~h}$ per day or $\leq 1 \mathrm{~h}$ per day, grouped according to asthma control. ${ }^{*} p<.05$ intergroup difference 
(A)

\section{Pha}

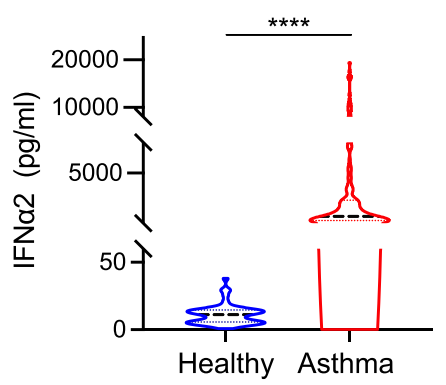

R848

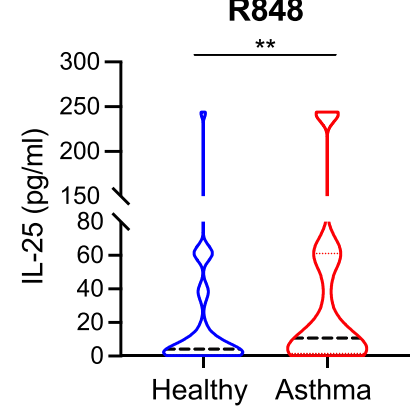

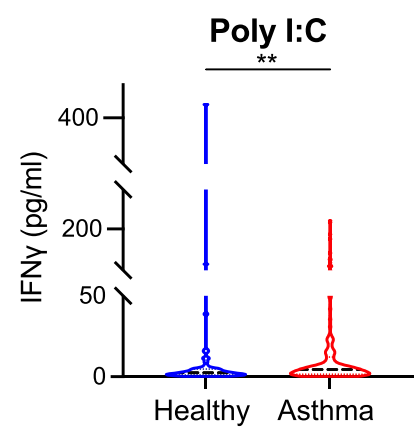

R848

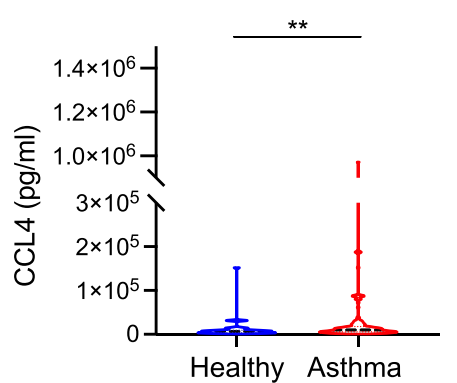

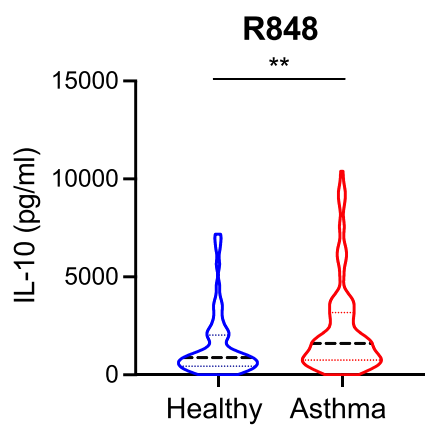
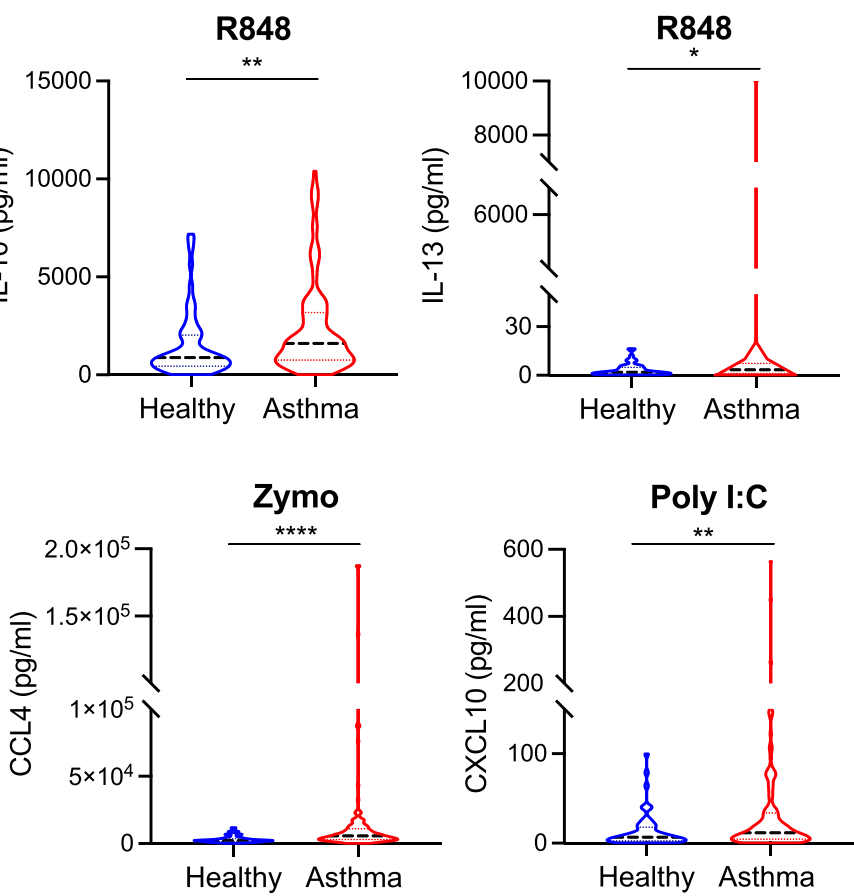

Poly I:C

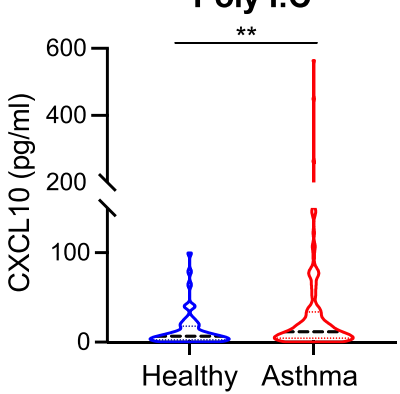

(B)
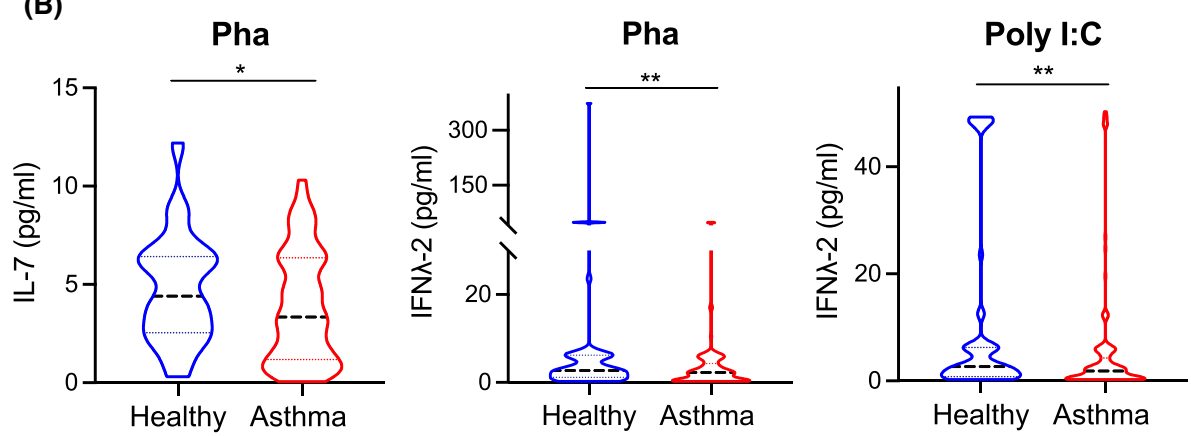

FIGURE 2 Increased (A) and decreased (B) cytokines under different stimulations in asthmatic preschoolers. Violin plots with median and quartiles visualizing cytokine levels. Asthma $n=140$, healthy $n=53$. PBMCs were cultured with different stimulants for 48 h. Cytokine levels were determined using a multiplex immunoassay. ${ }^{*} p<.05,{ }^{* *} p<.01$, and ${ }^{* * * *} p<.0001$ intergroup difference, respectively

vigorously physically active in the case group (30\% versus $25 \%$ of healthy subjects), whereas the control group showed more PA ( $\geq 3$ times of vigorous PA per week in $57 \%$ of controls versus $53 \%$ of cases). However, the differences in weekly PA were not statistically significant. A figure demonstrating the overlap of high TVA and low PA in asthmatic children is provided in Online Supplementary (Figure S1).

Children with poor asthma control were significantly more physically inactive $(75 \%$ never or occasionally physically active versus
$25 \% \geq 3$ times per week, Table 2 and Figure $1 \mathrm{~A}$ ). In contrast, there was a significantly higher proportion of physically active subjects in those with good asthma control (62\% of them with PA $\geq 3$ times per week and 18\% 1-2 times per week).

Daily TVA was slightly higher in children with uncontrolled asthma showing $81 \%>1 \mathrm{~h}$ TVA per day in the uncontrolled asthma group compared to $78 \%$ and $70 \%$ in the partly controlled asthma and controlled asthma groups, respectively (Figure 1B). However, no statistical significance was shown for this observation.

FIGURE 3 Impact of physical activity and TV attendance on unstimulated cytokines in asthmatic (A, B) and healthy (C, D) preschoolers. Violin plots with median and quartiles visualizing cytokine levels. Red and blue arrows indicate increase or decrease of cytokine levels with higher PA or TVA. (A) Asthma, physical activity: $\mathrm{n} / \mathrm{o}(n=42), 1-2 / \mathrm{w}(n=24), \geq 3 / \mathrm{w}(n=75)$. (B) Asthma, TV attendance: $\leq 1 \mathrm{~h} / \mathrm{d}(n=36)$, 1-3 h/d $(n=82), \geq 3 \mathrm{~h} /$ day $(n=22)$. (C) Healthy, physical activity: $\mathrm{n} / \mathrm{o}(n=13), 1-2 / \mathrm{w}(n=10), \geq 3 / \mathrm{w}(n=30)$. (D) Healthy, TV attendance: $\leq 1 \mathrm{~h} / \mathrm{d}(n=25), 1-3 \mathrm{~h} / \mathrm{d}(n=22), \geq 3 \mathrm{~h} /$ day $(n=6) .{ }^{*} p<.05,{ }^{* *} p<.01,{ }^{* * *} p<.001$ and ${ }^{* * * *} p<.0001$ intergroup difference, respectively. $\mathrm{n} / \mathrm{o}$ (never or occasionally), 1-2/w (once or twice per week), $\geq 3 / \mathrm{w}$ (3 or more times per week), $\leq 1 \mathrm{~h} / \mathrm{d}$ (less than one hour per day), 1-3 h/d (one to three hours per day), $\geq 3 \mathrm{~h} /$ day ( 3 or more hours per day) 
(A)

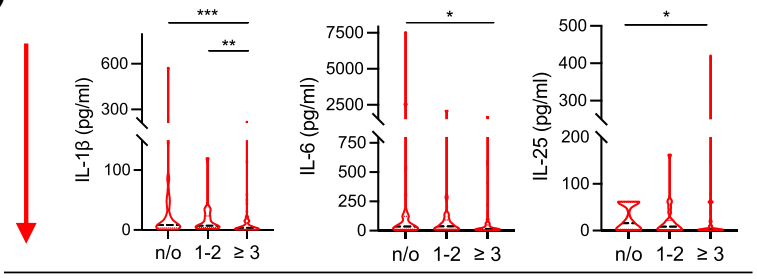

Physical activity per week

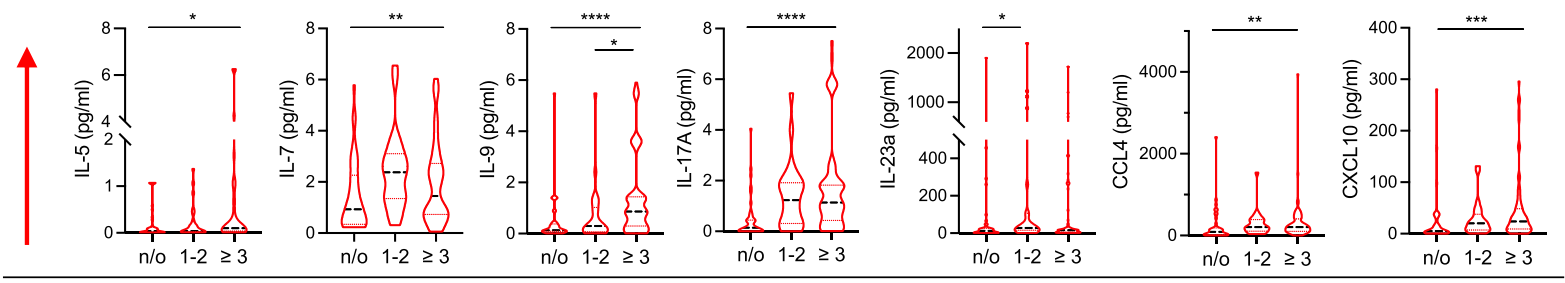

Physical activity per week

(B)

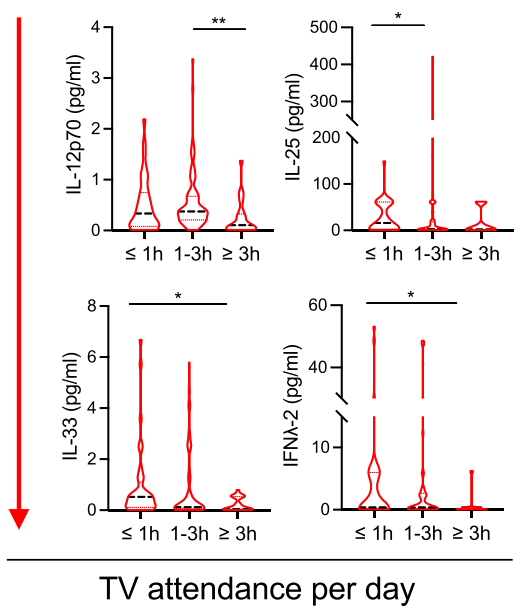

(C)

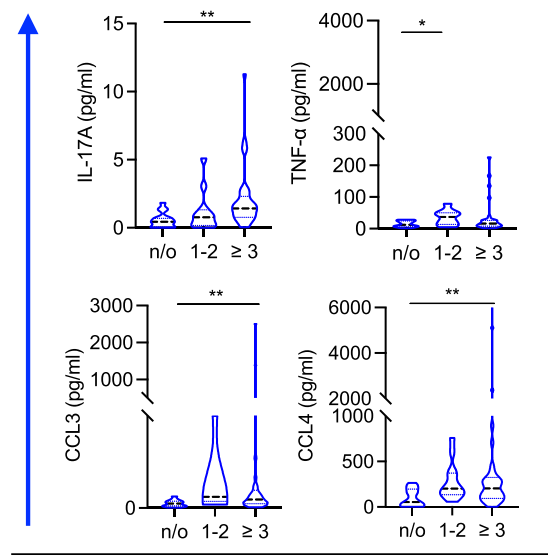

Physical activity per week

(D)
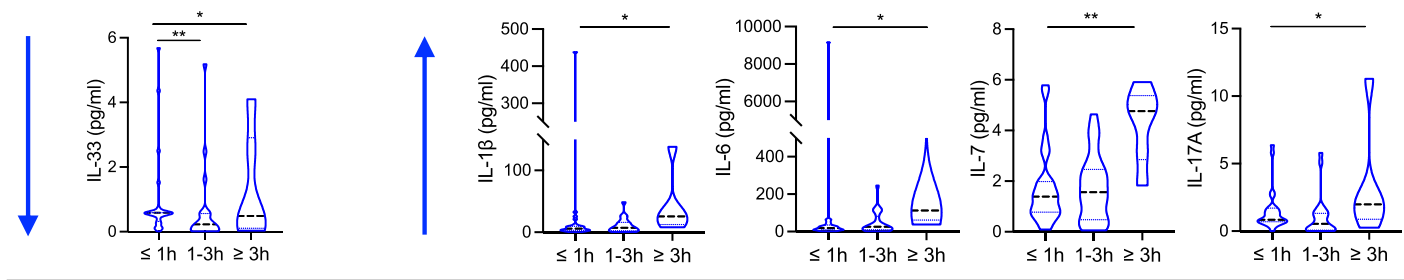

TV attendance per day

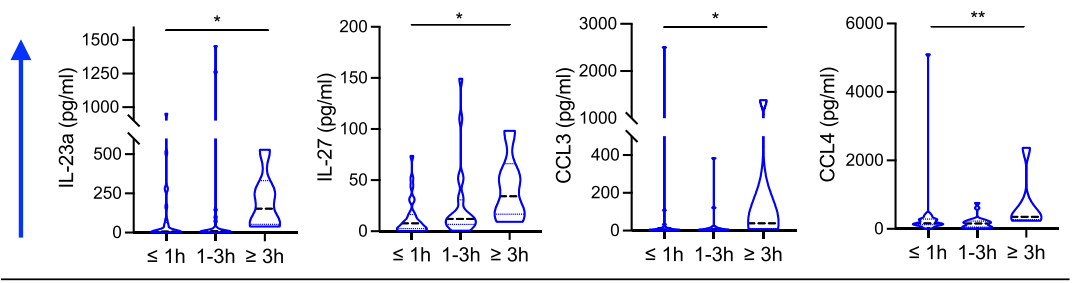

TV attendance per day 
(A)

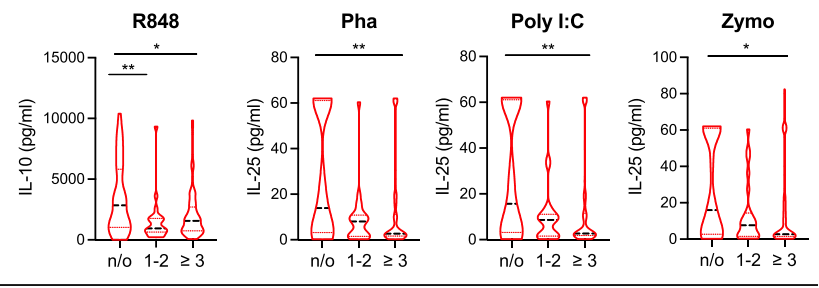

Physical activity per week

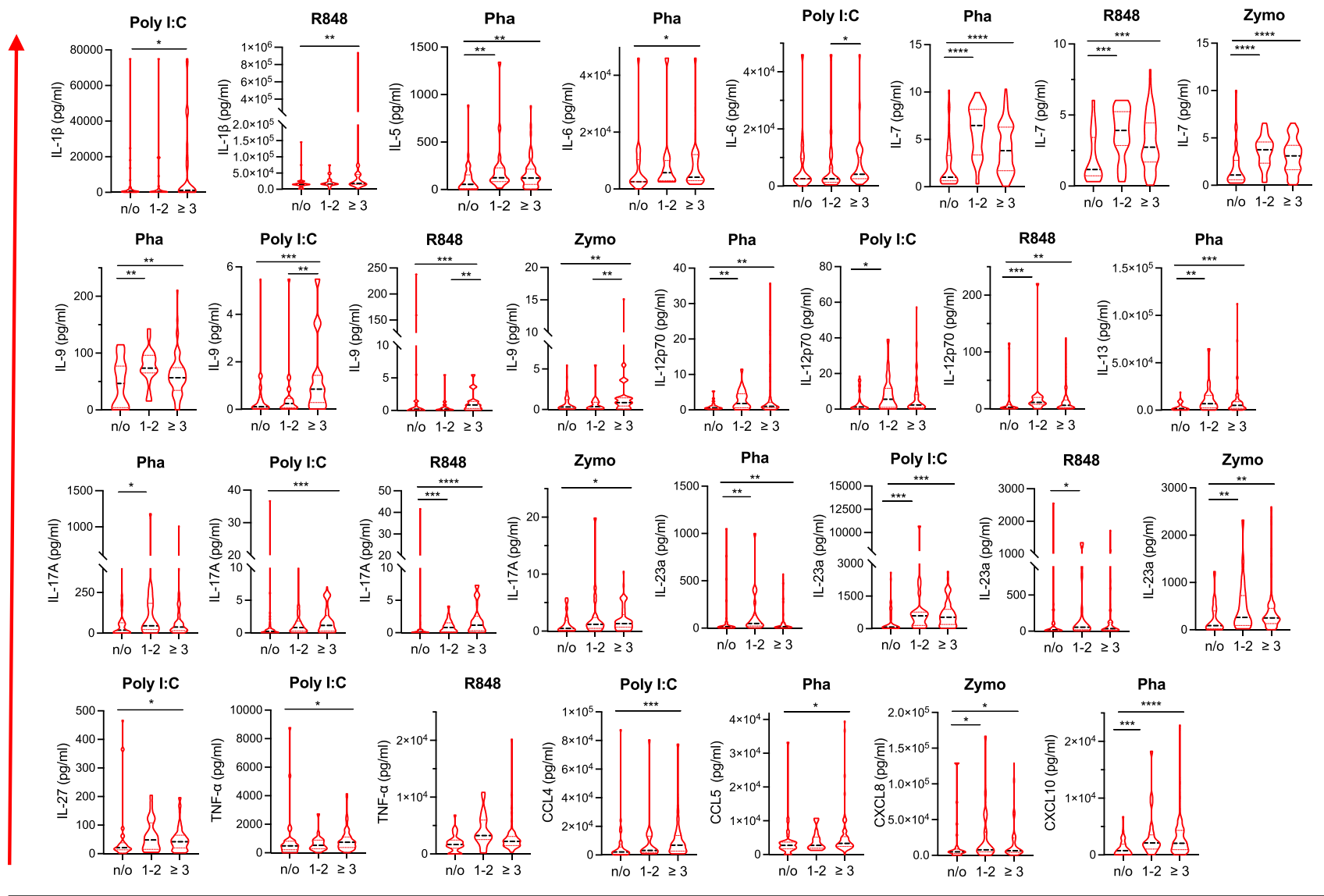

Physical activity per week

(B)

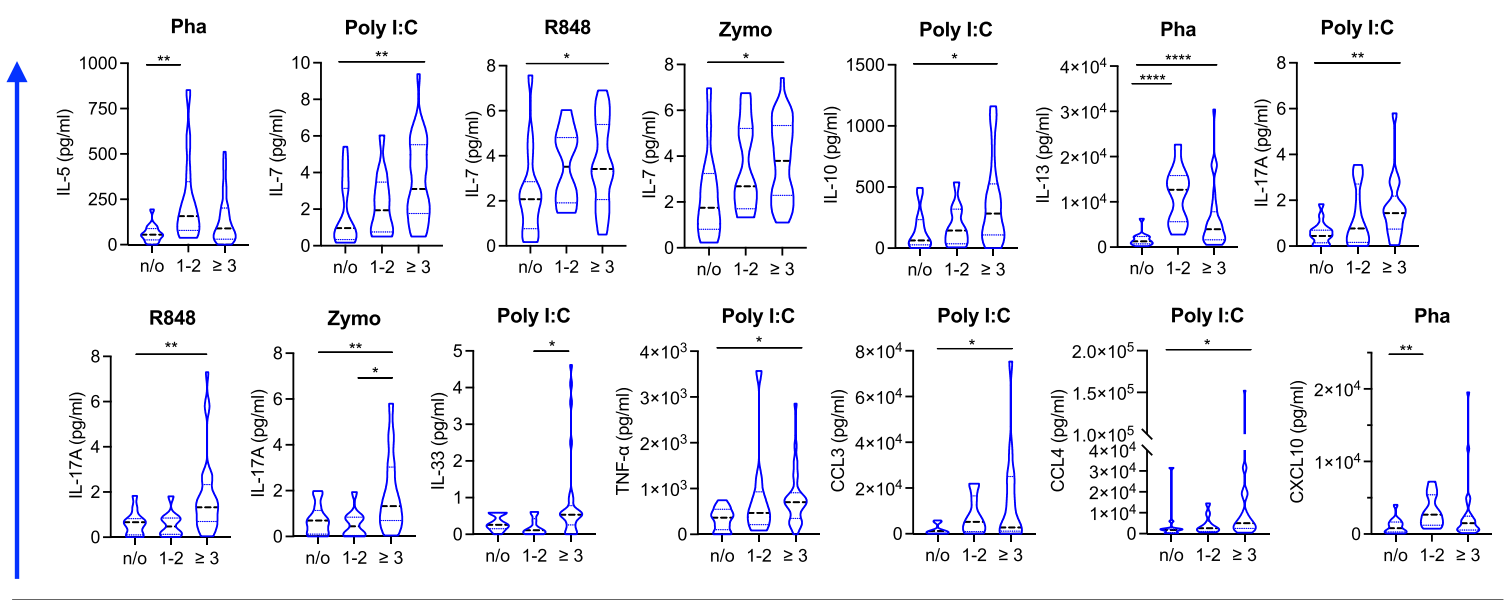

Physical activity per week 
FIGURE 4 Impact of physical activity on PBMC cytokine response in asthmatic (A) and healthy (B) preschoolers. Violin plots with median and quartiles visualizing cytokine levels. Red and blue arrows indicate increase or decrease of cytokine levels with higher PA. (A) Asthma, physical activity: $\mathrm{n} / \mathrm{o}(n=42), 1-2 / \mathrm{w}(n=24), \geq 3 / \mathrm{w}(n=75)$. (B) Healthy, physical activity: $n / o(n=13), 1-2 / w(n=10), \geq 3 / w(n=30)$. ${ }^{*} p<.05,{ }^{* *} p<.01,{ }^{* * *} p<.001$, and ${ }^{* * * *} p<.0001$ intergroup difference, respectively. n/o (never or occasionally), 1-2/w (once or twice per week), $\geq 3 / \mathrm{w}$ ( 3 or more times per week)

\section{3 | Cytokine levels under different stimulations in asthmatic preschoolers}

No significant differences could be observed for baseline cytokine levels between healthy and asthmatic subjects, as displayed in Figure S2.

Asthmatic children showed high numbers of cytokines increased in response to various stimuli compared to healthy subjects (Figure 2A). Particularly, R848 and poly I:C were the stimulants that induced IL-10, IL-13, IL-25, IFN $\gamma$, CCL4, and CXCL10, while stimulation with PHA and zymo induced IFN $\alpha 2$ and CCL4, respectively.

In contrast, only IL-7 and IFN $\lambda$-2 were found to be lower in asthmatic in comparison with healthy subjects after stimulation with PHA and poly I:C (Figure 2B). Cytokines that showed no significant difference between the two groups after polyclonal stimulation are found in Online Supplementary (Figures S3-S6).

\subsection{Effect of physical activity and TV attendance on unstimulated cytokine levels}

In unstimulated conditions, levels of IL-5, IL-7, IL-9, IL-17A, IL-23A, CCL4, and CXCL10 were significantly higher, and levels of IL-1 $1 \beta$, IL-6, and IL-25 significantly lower in asthmatic children who were more physically active compared to those who never or only occasionally exercised (Figure 3A). In healthy subjects, increased cytokine levels with higher weekly PA could only be observed for IL-17A, TNF- $\alpha$, CCL3, and CCL4 (Figure 3C).

Again, in unstimulated conditions, high daily TVA was associated with decreased levels of IL-12p70, IL-25, IL-33, and IFN $\lambda$-2 in asthmatic children (Figure $3 \mathrm{~B}$ ). Healthy children with a more sedentary behavior exhibited increased IL-1 $\beta$, IL-6, IL-7, IL-17A, IL-23A, IL-27, CCL3, and CCL4, whereas their IL-33 levels decreased (Figure 3D). Cytokines that showed no significant difference in baseline levels between the two groups according to PA and TVA are found in Online Supplementary (Figures S7 and S8).

\section{5 | Effect of physical activity on cytokine levels of polyclonally stimulated PBMCs}

Asthmatic children with high PA showed elevated levels of IL-1 $\beta$, IL5, IL-6, IL-7, IL-9, IL-12p70, IL-13, IL-17A, IL-23A, IL-27, TNF- $\alpha$, CCL4, CCL5, CXCL8, and CXCL10 in response to stimulation with PHA, poly I:C, R848, and zymo (Figure 4A). A significant decrease was only observed for IL-10 in PBMCs stimulated with R848 and for IL25 after stimulation with PHA, poly I:C, and zymo.
In healthy children, high PA was associated with increased levels of IL-5, IL-7, IL-10, IL-17A, IL-33, TNF- $\alpha$, CCL3, CCL4, and CXCL10 after PBMC stimulation with PHA, poly I:C, R848, and zymo (Figure 4B). Cytokines that showed no significant difference between asthmatic and healthy subjects according to their PA level are found in Online Supplementary (Figures S9-S12).

\subsection{Effect of TV attendance on stimulated cytokine levels of polyclonally stimulated PBMCs}

There were higher levels of IL-10 in response to stimulation with PHA and R848 in asthmatic children with high daily (Figure 5A). Elevated levels were also observed for CXCL8 after stimulation with zymo. A decrease in secretion of IL-1 $\beta$, IL-6, IL-12p70, IL-13, IL-17A, IL-25, IL-33, IFN $\alpha 2$, IFN $\gamma$, and CXCL10 was observed in response to stimulation with PHA, poly I:C, R848, and zymo.

Daily long-time TVA in healthy children was associated with increased levels of IL-7, IL-12p70, IL-23A, TNF- $\alpha$, IFN $\alpha 2$, IFN $\gamma$, CCL3, CCL5, and CXCL10 in PBMC cultures stimulated by PHA, poly I:C, R848, and zymo. A slight reduction in IL-33 and CXCL8 levels was observed for healthy subjects with longer TVA after stimulation with zymo and PHA, respectively. Cytokines that showed no significant difference between asthmatic and healthy subjects according to their daily TVA are found in Online Supplementary (Figures S13-S16).

\section{7 | Correlation of unstimulated and stimulated cytokines in preschoolers with low physical activity and high TV attendance}

Asthmatic children with no or only occasional PA and / or TVA $\geq 3 \mathrm{~h}$ per day showed high correlations of proinflammatory cytokines (Figure 6A,B), whereas barely any clusters of correlation could be identified in healthy children of the same PA and TVA groups (Figure 6C,D).

Cytokines that showed particularly high correlations in asthmatics include IL-1 $\beta$, IL-5, IL-6, IL-7, IL-9, IL-10, IL-12p70, IL-13, IL-23A, IL-27, TNF- $\alpha$, IFN $\alpha 2$, IFN $\gamma$, CCL3, CCL4, and CXCL10. Mostly, these highly correlating cytokines were either measured in unstimulated conditions or after PBMC stimulation with PHA, poly I:C, and R848.

\section{4 | DISCUSSION}

In the present study, we investigated the immunological effects of PA and asthma control. Children with controlled asthma engaged in vigorous PA considerably more often compared to those with 
(A)
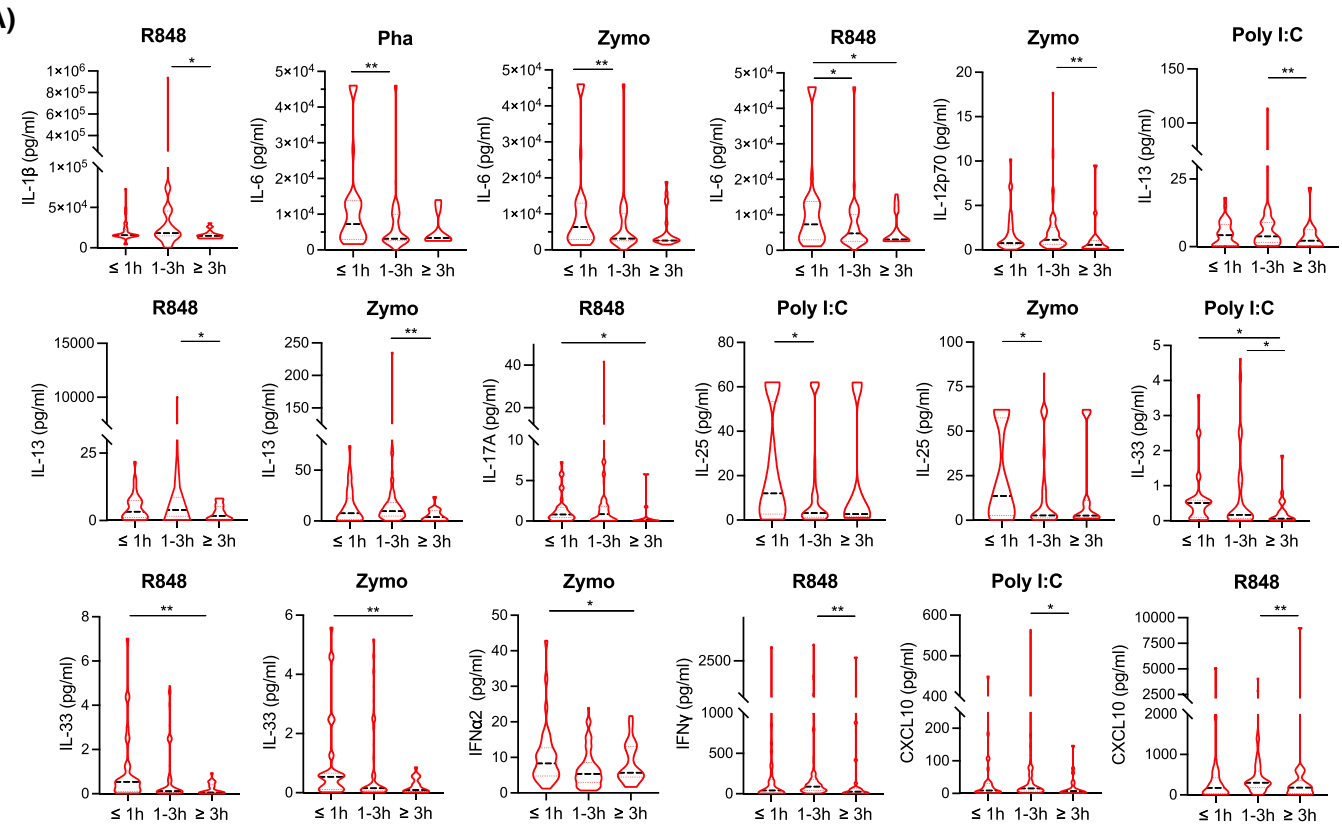

TV attendance per day

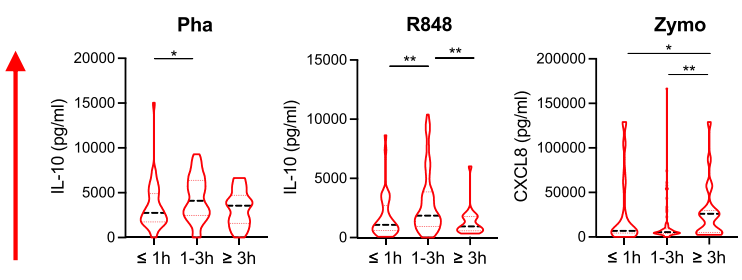

TV attendance per day

(B)

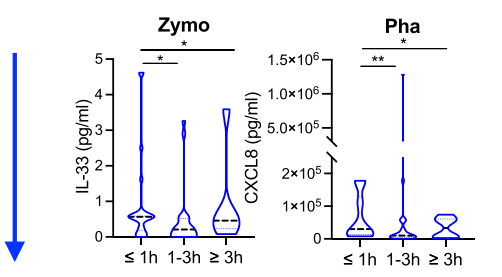

TV attendance per day

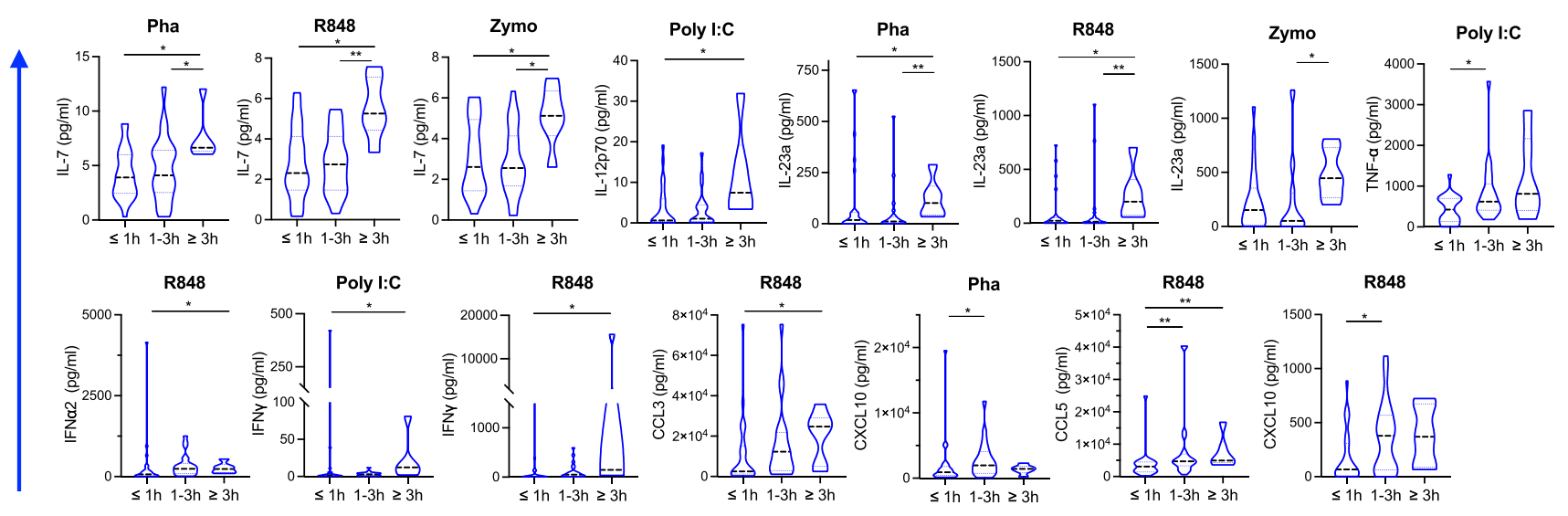


FIGURE 5 Impact of TV attendance on PBMC cytokine response in asthmatic (A) and healthy (B) preschoolers. Violin plots with median and quartiles visualizing cytokine levels. Red and blue arrows indicate increase or decrease of cytokine levels with higher TVA. (A) Asthma, TV attendance: $\leq 1 \mathrm{~h} / \mathrm{d}(n=36), 1-3 \mathrm{~h} / \mathrm{d}(n=82), \geq 3 \mathrm{~h} /$ day $(n=22)$. (B) Healthy, TV attendance: $\leq 1 \mathrm{~h} / \mathrm{d}(n=25), 1-3 \mathrm{~h} / \mathrm{d}(n=22), \geq 3 \mathrm{~h} / \mathrm{day}$ $(n=6) .{ }^{*} p<.05,{ }^{* *} p<.01,{ }^{* * *} p<.001$ and ${ }^{* * * *} p<.0001$ intergroup difference, respectively. $\leq 1 \mathrm{~h} / \mathrm{d}$ (less than one hour per day), $1-3 \mathrm{~h} / \mathrm{d}$ (one to three hours per day), $\geq 3 \mathrm{~h} /$ day (3 or more hours per day)

partially controlled or uncontrolled asthma. In addition, asthmatic preschoolers reported more daily TV hours compared to their healthy peers. As a general finding, physically active asthmatics expressed higher levels of various cytokines in PBMC cultures under both unstimulated conditions and stimulation with different polyclonal stimulants, while long daily TVA was associated with an overall decrease in cytokine levels.

Asthmatics are commonly perceived as being more physically inactive in comparison with healthy individuals. ${ }^{3}$ Significantly less TV hours in healthy children shown in the present study, which we interpret as less sedentary behavior, supports this notion. One explanation for this behavior can be described as a vicious circle: in fear of experiencing exercise-induced dyspnea, the parents or the child might consciously or unconsciously restrict his or her practice of PA. ${ }^{33}$ Of course, such avoidance behavior particularly affects subjects with severe and/or uncontrolled asthma. ${ }^{3,13}$ Our study supports the importance of PA in asthma control, showing that children with controlled asthma are engaged in PA significantly more often than their peers with uncontrolled asthma.

Although there was a significant difference between controlled and uncontrolled asthma, we did not find any significant difference regarding the weekly amount of PA between asthmatic and healthy children in the present study. The impact of asthma diagnosis on sports in childhood and adolescence is generally accepted. The AIRE study demonstrated that $30 \%$ of asthmatic children felt limited in their physical activities. ${ }^{34}$ It was also reported that a majority of asthmatic children perceived the inability to participate in sports as the worst thing about their asthma. ${ }^{35}$ So far, studies comparing the level of PA in asthmatic and non-asthmatic children have shown controversial results. ${ }^{3}$ Whereas Firrincieli et al. found that asthmatic children are less physically active, ${ }^{33}$ others suggested their level of PA does not differ from healthy children, ${ }^{5,13,35}$ or that they are even more active. ${ }^{36}$ Factors that could make for this inconsistency could be the asthma diagnosis criteria used in this age group, awareness of the doctors for suggesting PA in the cohort, regional cultural differences as well as the instruments used to quantify PA.

Exercise is known to enhance the health-related quality of life in asthmatics not only by improving aerobic capacity, but also by reducing dyspnea, the intensity of exercise-induced bronchoconstriction, the dose of inhaled corticosteroids, and exacerbation of their asthma. ${ }^{13,37}$ Therefore, the American College of Sports Medicine and the American Thoracic Society endorse prescription of PA for all asthmatic subjects. ${ }^{6}$ Guidelines focused on physical activity for pediatric asthma patients are lacking. A recommendation to exercise on a regular basis for children with controlled asthma is made by the Global Initiative for Asthma (GINA). ${ }^{38}$ In addition, there has been substantial research on the use of exercise to treat asthma, which also proved to be safe and beneficial for pediatric asthmatic subjects. ${ }^{39}$ Bonini et al. conducted a study with Italian Olympic athletes that may give young asthmatics every reason to support their PA. The authors reported that adequately diagnosed and treated asthmatic athletes can compete at the highest level. ${ }^{7,40}$

The present study also investigated in vitro immune response of asthmatic and healthy children by analyzing cytokines produced from PBMC that may contribute to asthmatic inflammation in allergic and nonallergic asthma. ${ }^{15}$ Baseline cytokine levels of unstimulated PBMC did not differ between the two groups. Assessment of unstimulated cytokine levels in different levels of PA or TVA (Figure 3) revealed that cytokine response to regular physical exercise is more pronounced in asthmatic children. This may in part be interpreted as the result of the underlying systemic inflammation due to their asthma. For our investigation of immune responses in PBMC cultures, we chose four stimulants: Phytohemagglutinin (PHA) acts as a mitogen that leads to a polyclonal immune activation, poly I:C, and R848 both mimic respiratory viral infections and zymosan (zymo) mimics immune response to a fungal infection. Compared to healthy subjects, asthmatic preschoolers show higher cytokine levels particularly after stimulation poly I:C and R848, indicating a strong response in case of respiratory virus contraction. Regular exercise is further known to have anti-inflammatory effects, which most likely play an important role in its ability to reduce the risk of chronic metabolic and cardiorespiratory diseases. ${ }^{23,41}$ The three main mechanisms that are thought to lead to the anti-inflammatory effects of regular exercise are a reduction in visceral fat mass (leading to a decrease in proinflammatory adipokines, eg, TNF- $\alpha$ ), an increased production of anti-inflammatory cytokines from contracting skeletal muscle (myokines; eg, IL- 6 leading to a subsequent rise in antiinflammatory IL-10 and IL-1-RA) and a reduction of Toll-like receptor expression on monocytes and macrophages. ${ }^{41}$

Our results show that a higher amount of weekly vigorous PA is associated with a great number of elevated cytokine levels in response to all four stimulants, which could indicate an immune system prepared for responding strongly in case of infection. PA is known to affect both innate and acquired immune response in various ways. As such, an increase in NK cell numbers and NK cell cytotoxicity, as well as a decrease in T-cell functionality, has been described in response to exercise. ${ }^{22,24}$ An imbalance between Th1/ Th2/Th17 cells and their control by Treg cells can play a crucial role in asthma development, while different phenotypes show distinct immunological patterns. ${ }^{15,18-20}$ Type 2 inflammation is linked to the most common asthma phenotype and allergic asthma. ${ }^{15,19}$ Type 2 cytokines (eg, IL-4, IL-5, IL-9, and IL-13), which are activated by allergen exposure, can cause airway hyperresponsiveness by contraction of smooth muscles, mucus production, eosinophil activation, and an induction of allergen-specific IgE by B lymphocytes. ${ }^{19,42}$ Those type 2 cytokines can also be secreted by innate lymphoid cells (ILC2), 
(A)

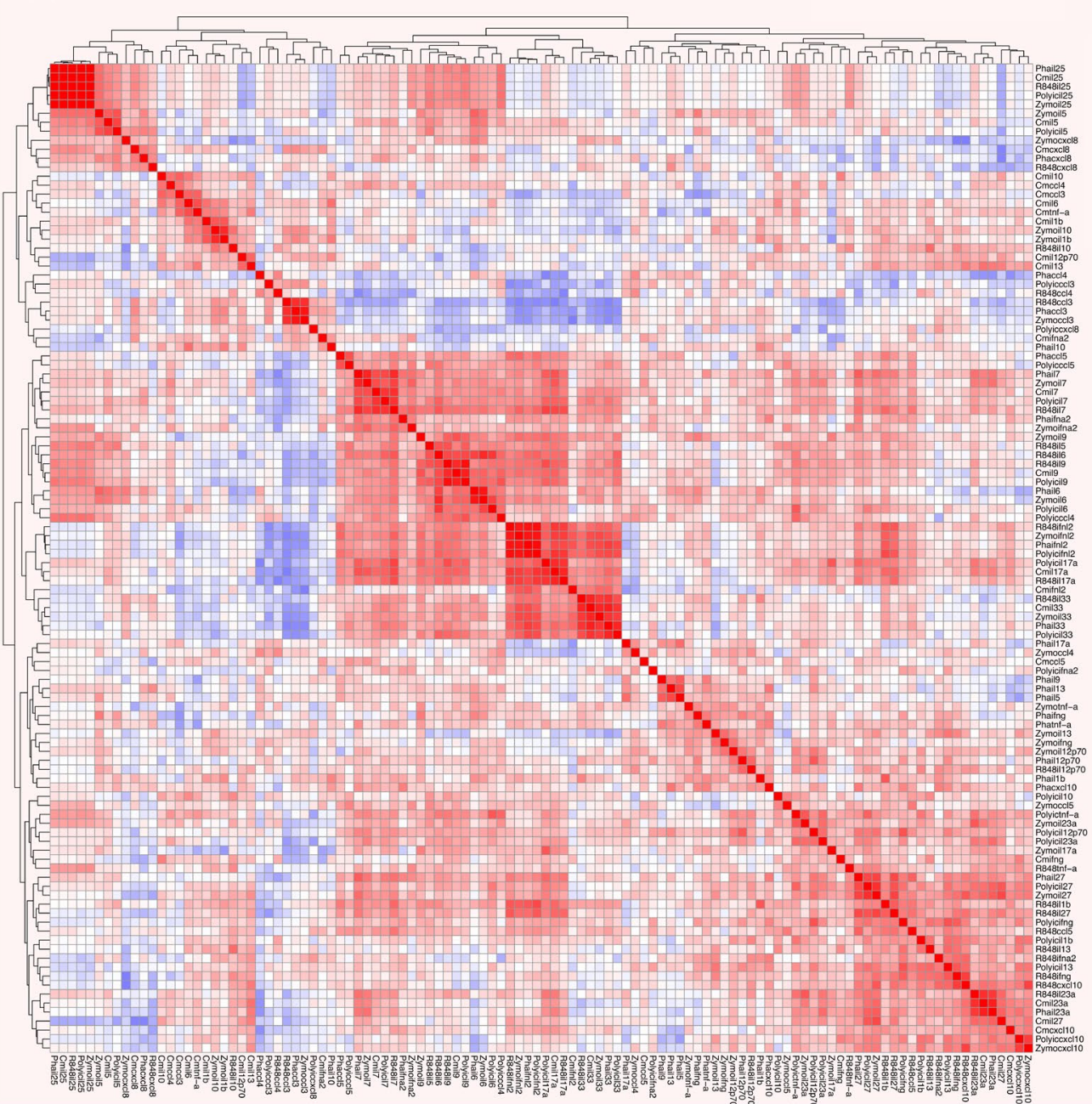

(B) TVA $\geq 3 \mathrm{~h} / \mathrm{d}$

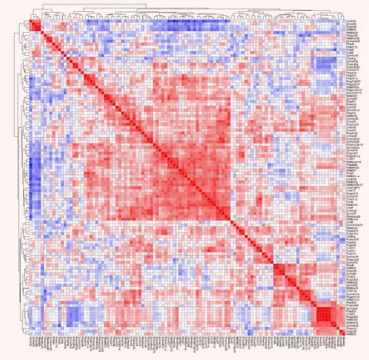

(C)

PA n / o

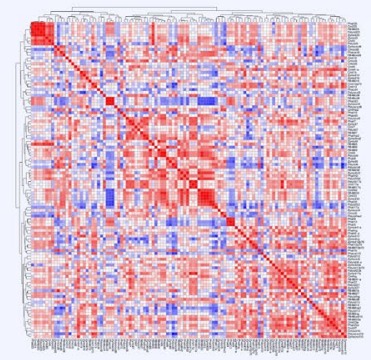

(D)

\section{TVA $\geq 3 h / d$}

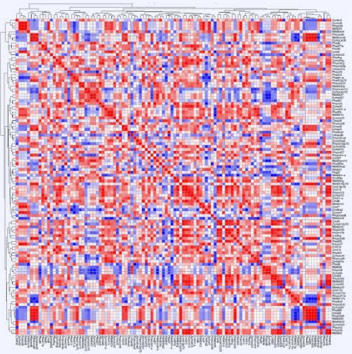

(E)
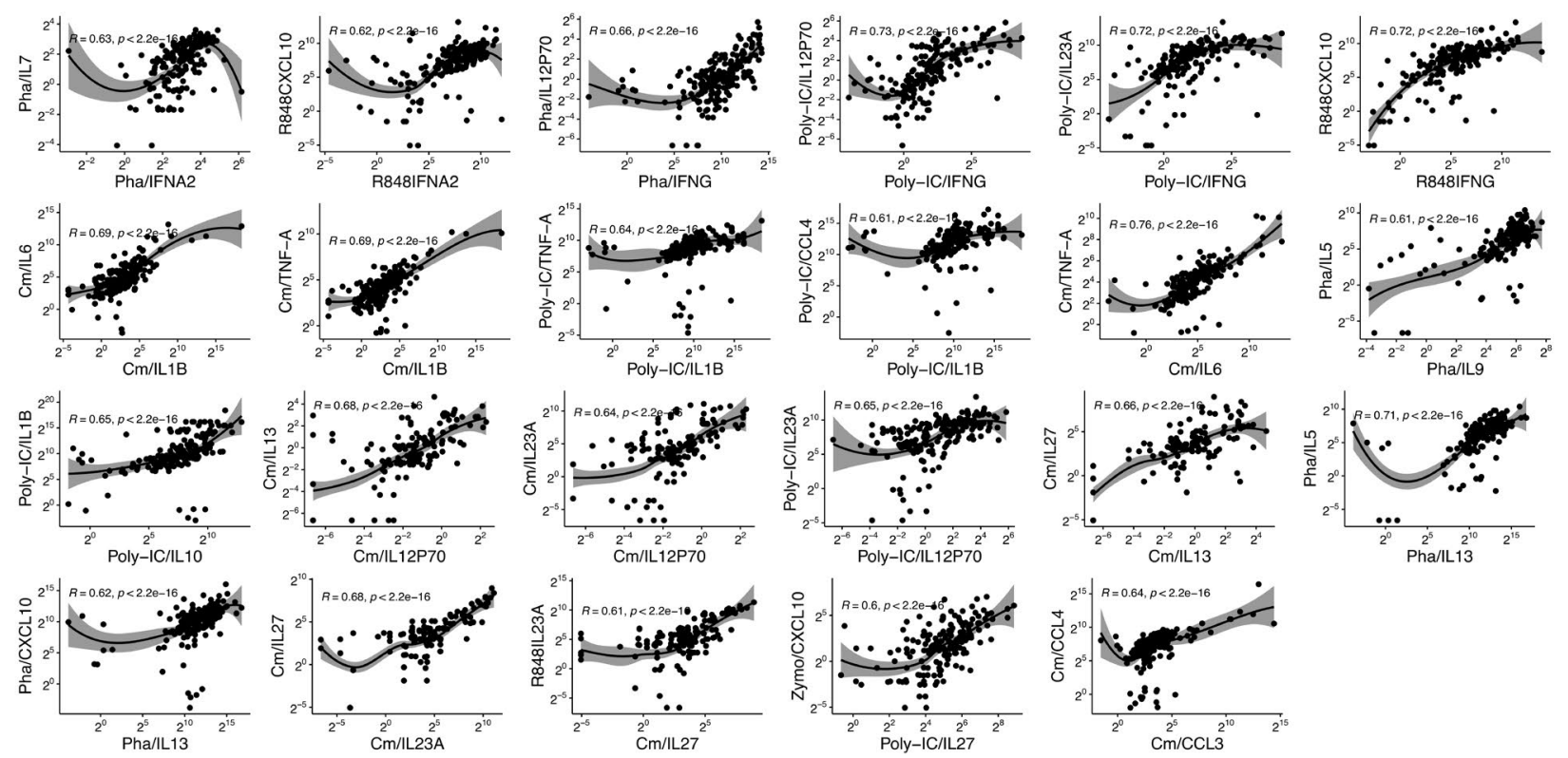

FIGURE 6 Correlations between cytokines in asthmatic and healthy preschoolers with low weekly physical activity (A, C) and high daily TV attendance (B, D). Spearman correlation heat maps visualizing correlations between measured cytokines in both asthmatic (A, B) and healthy $(C, D)$ preschoolers. Red and blue squares indicate positive $(0$ to +1$)$ and negative $(0$ to -1$)$ correlations, respectively. (E) Spearman correlation scatter plots showing significant correlations between cytokines 
after stimulation by epithelial IL-25 and IL-33, which are generated by impaired airway epithelial cells in asthmatic subjects. ${ }^{15,19,43}$

Due to ethical limitations, immune response to PA in children has been poorly investigated. ${ }^{24}$ In atopic individuals, PA may cause further Th2 polarization, leading to more severe allergic symptoms or exercise-related symptoms. ${ }^{24}$ However, it has also been shown that exercising on a regular basis induced beneficial changes in allergic subjects, such as a reduction in proinflammatory cytokines (eg, (L- $4^{44}$ ) and a switch to a type 1 profile, which in turn may reduce allergic inflammation. ${ }^{24}$ Studies in murine asthma models reported an enhancement of Treg responses to aerobic exercise. ${ }^{25}$

In the present study, we found some type 2 cytokines (IL-5, IL-9, IL-13, and CCL5) to be upregulated in asthmatic preschoolers with a higher level of weekly PA, whereas IL-25 levels were significantly lower in more physically active individuals. The increase is not only found in type 2 cytokines, because regarding type 1- and type 17-related cytokines, IL-12B, IL-17, IL-27, TNF- $\alpha$, and CXCL10 were found to be upregulated in asthmatic preschoolers with high weekly PA, supporting the readiness to produce both type 1 , type 2 , and type 17 cytokines in response to various stimuli. These findings can be perceived as immunological fitness without any skew to a certain subtype observed by extensive PA. However, it may also implicate that very high levels of PA might lead to a hyperinflammatory state, which would not be beneficial in asthmatic individuals.

Apart from the Th1/Th2 imbalance predominantly found in allergic asthmatics, a disequilibrium in Th17 and Treg cells has been noted in nonallergic asthmatic subjects with neutrophilic airway inflammation. ${ }^{15,18} \mathrm{IL}-17$, produced by Th17 cells, may be upregulated in these patients, while Treg functions are inhibited in children with asthma. ${ }^{45,46}$ This Th17/Treg imbalance was shown to be closely associated with asthma severity and steroid resistance. ${ }^{15,47}$ The present study illustrated IL-17A is upregulated in asthmatic preschoolers with high PA. As Th1 and Th17 cells, which produce IL-17A, are dominant in neutrophilic asthma, ${ }^{15}$ it could be hypothesized that PA might not have a beneficial effect in every asthma phenotype. However, we also observed a decrease of IL- 6 and IL-1 $\beta$ levels in highly physically active asthmatic preschoolers. IL- 6 is required for Th17 differentiation and IL-1 $\beta$ promotes Th17 cell-dependent inflammation, ${ }^{15}$ both leading to a disequilibrium of Th17/Treg cells (toward Th17). A fall in IL- 6 and IL- $1 \beta$ levels may therefore positively impact the disbalance in such patients.

Longer TVA represent a longer indoor stay and less outdoor allergen and air pollutant exposure and a less physically active condition. Our results demonstrate an overall decrease in cytokine levels in asthmatic preschoolers with high daily TVA, indicating weaker immune responses to various stimuli (bacterial, viral, or fungal) compared to subjects with less daily TV hours. However, the Th2-related cytokines IL-25, IL-33, and IL-13, all playing a major role in allergic asthma, were found to be downregulated in asthmatic children with high TVA. Since they are epithelial cytokines, longer stay indoors, and less epithelial cell activation can be one of the reasons for this. It could therefore be argued that high TVA might have a positive impact on the epithelial cell alarmins that may be the initiators of type
2 inflammation. Furthermore, IFN $\gamma$, which has previously been found to be associated with non-eosinophilic asthma and steroid-resistant asthma, ${ }^{15}$ was significantly downregulated in asthmatic preschoolers with high TVA.

While some of these results indicate a potential positive influence of TVA on immunological reactions in asthmatic preschoolers, our data also show that asthmatic subjects with low weekly PA and / or high daily TVA exhibited highly positively correlating proinflammatory cytokines under stimulated, but also unstimulated conditions, suggesting an overall proinflammatory state in those individuals. Furthermore, the association between a sedentary lifestyle and obesity is kept in mind. The worldwide increase in asthma prevalence occurred together with an increase in obesity and a sedentary lifestyle. ${ }^{39}$ A number of studies reported an association between obesity and childhood asthma; however, the causality is not clear. ${ }^{5,48}$

Our study has a number of limitations. Firstly, the PreDicta cohort is moderate in size, however, performing cell cultures in a standard way and measurement of many cytokines in all these patients and controls should be appreciated. The lack of objective criteria makes the diagnosis of asthma more difficult in preschoolers. In addition, parameters such as weekly PA or daily TVA were collected from questionnaires based on parental reports. Even though short-term parental reports were shown to be accurate, ${ }^{49}$ recall bias might be a source of error. For example, strictly interpreting daily TV hours as sedentary behavior can lead to the wrong assumption that a subject with high daily TVA cannot simultaneously be vigorously physically active more than 3 times a week. Our findings of lower PA with poor asthma control are prone to reverse causation bias. That is, while we suppose that poor asthma control leads to the observed decrease in PA, the association might as well be at least in part reversed, that is, lower PA leads to poor asthma control. Observational studies commonly face the problem of reverse causation bias because causality cannot usually be assessed from observational data alone. Therefore, for the detailed assessment of causal inference in the case of PA and asthma control, controlled studies are required. It is unclear whether the differences observed in cytokine levels actually result from the physical activity status of the subjects. Many factors, such as genetics, type of asthma, asthma control, current medication, infections, immunizations, various exposures, and diet, can influence cytokine levels. For example, it can be well argued that the decrease in IL-25 levels in asthmatic children with high weekly PA might be due to their well-controlled asthma and the regular use of asthma medication. 120 out of 140 asthmatic children used inhaled corticosteroids (ICS). Although the usage of steroids may influence peripheral blood cytokine responses, it was not possible to elaborate the effect of ICS on the cytokine responses in the present study. It has to be additionally considered and needs further studies whether children staying indoors with increased TVA are having less exposure to environmental pollutants and outdoor allergens. Furthermore, low PA and high TVA are more likely to be results of uncontrolled asthma, which will be addressed elsewhere. Lastly, it would have added great value to this study if BMI values 
had been assessed. Since obesity is a proinflammatory state with elevated hormones (eg, leptin), cytokines (eg, IL-1, IL-6, and TNF- $\alpha$ ), and chemokines, ${ }^{39,50}$ our data could be influenced by this comorbidity. As obesity certainly plays a role in the development and severity of asthma, ${ }^{39,51}$ collection of BMI data, and whenever possible assessment markers of metabolic dysfunction (eg, hyperglycemia, hyperinsulinemia), ${ }^{51}$ should be standard procedure in the future pediatric asthma cohorts.

In conclusion, our work shows an association of limited PA with poor asthma control and that both PA and TVA possibly impact systemic immune response and immune and inflammatory thresholds in asthmatic preschoolers. Our findings of good immune response to viral, bacterial, and fungal stimulation in physically active asthmatic children support the common recommendation that regular PA should be encouraged in this patient group, while good asthma control is essential. The high cytokine levels observed in association with high PA might also implicate hyperinflammation, indicating that the recommendation for PA should include individual assessment to find an optimum level of PA, which might be more moderate. The fitness and readiness of the immune system to secrete cytokines are becoming more and more important, with the recent knowledge in COVID-19, for example, in timely release of antiviral interferons. ${ }^{52,53}$ However, an exaggerated cytokine release (eg, IL$1 \mathrm{~B}$ and IL-6) may be part of a detrimental inflammatory response as observed in the cytokine storm of COVID-19. Further studies are required to investigate the roles of controlled PA or "hyper" PA on inflammatory responses. Finally, it is of great importance not to forget about the association of physical inactivity, poor physical cardiovascular fitness, and obesity-all of which threatens a child's health and well-being.

\section{ACKNOWLEDGMENTS}

This research was funded by PreDicta, EU 7th framework programme, Contract No: HEALTH-F2-2010-260895 and Swiss National Science Foundation.

\section{CONFLICT OF INTEREST}

DJM, CL, SF, YG, AG, TJ, WK, HL, BS, GT, MV, LZ, NZ, and MA have nothing to disclose. PX reports personal fees from Uriach, Novartis, Néstle, and Nutricia outside the submitted work. MP reports grants from University of Athens during the conduct of the study and personal fees from Aimmune Therapeutics, grants from Hellenic Society of Allergy and Clinical Immunology outside the submitted work. NP reports personal fees from Novartis, Nutricia, HAL, MENARINI/FAES FARMA, SANOFI, MYLAN/MEDA, BIOMAY, AstraZeneca, GSK, MSD, ASIT BIOTECH, Boehringer Ingelheim, grants from Gerolymatos International SA, Capricare outside the submitted work. CA reports grants form Allergopharma, Idorsia, Swiss National Science Foundation, Christine Kühne-Center for Allergy Research and Education, European Commission's Horison's 2020 Framework Programme, Cure, Novartis Research Institutes, Astra Zeneca, SciBase advisory role and is on the advisory board for
Sanofi/Regeneron, GSK, Novartis. CB received honoraria from ALK, Astra-Zeneca, GSK, Novartis, and Sanofi outside of this work.

\section{ORCID}

Debbie J. Maurer (D) https://orcid.org/0000-0002-8543-1998

Chengyao Liu (D) https://orcid.org/0000-0002-0056-9032 Paraskevi Xepapadaki (D) https://orcid.org/0000-0001-9204-1923

Claus Bachert (D) https://orcid.org/0000-0003-4742-1665

Susetta Finotto (D) https://orcid.org/0000-0001-7623-7977

Ya-Dong Gao (D) https://orcid.org/0000-0003-1251-7608

Tuomas Jartti (D) https://orcid.org/0000-0003-2748-5362

Walter Kistler (iD https://orcid.org/0000-0002-9289-6953

Marek Kowalski (D) https://orcid.org/0000-0002-8442-2774

Ge Tan (D) https://orcid.org/0000-0003-0026-8739

Luo Zhang (D) https://orcid.org/0000-0002-0910-9884

Mübeccel Akdis (D) https://orcid.org/0000-0003-0554-9943

Nikolaos G. Papadopoulos (D) https://orcid.

org/0000-0002-4448-3468

Cezmi A. Akdis (D) https://orcid.org/0000-0001-8020-019X

\section{REFERENCES}

1. Asher I, Pearce N. Global burden of asthma among children. Int J Tuberc Lung Dis. 2014;18(11):1269-1278.

2. Papadopoulos NG, Arakawa H, Carlsen $\mathrm{KH}$, et al. International consensus on (ICON) pediatric asthma. Allergy. 2012;67(8):976-997.

3. Welsh L, Roberts RG, Kemp JG. Fitness and physical activity in children with asthma. Sports Med. 2004;34(13):861-870.

4. Pearce N, Aït-Khaled N, Beasley R, et al. Worldwide trends in the prevalence of asthma symptoms: phase III of the International Study of Asthma and Allergies in Childhood (ISAAC). Thorax. 2007;62(9):758-766.

5. Berntsen S, Carlsen KC, Anderssen SA, et al. Norwegian adolescents with asthma are physical active and fit. Allergy. 2009;64(3):421-426.

6. Lucas SR, Platts-Mills TA. Physical activity and exercise in asthma: relevance to etiology and treatment. J Allergy Clin Immunol. 2005;115(5):928-934.

7. Del Giacco SR, Manconi PE, Del Giacco GS. Allergy and sports. Allergy. 2001;56(3):215-223.

8. World Health Organization (WHO). Global Action Plan on Physical Activity 2018-2030: More Active People for a Healthier World. World Health Organization (WHO); 2018.

9. Pedersen BK, Saltin B. Exercise as medicine - evidence for prescribing exercise as therapy in 26 different chronic diseases. Scand J Med Sci Sports. 2015;25(Suppl 3):1-72.

10. Bull FC, Al-Ansari SS, Biddle S, et al. World Health Organization 2020 guidelines on physical activity and sedentary behaviour. $\mathrm{Br} \mathrm{J}$ Sports Med. 2020;54(24):1451-1462.

11. World Health Organization (WHO). Global Recommendations on Physical Activity for Health. World Health Organization (WHO); 2010.

12. Global Initiative for Asthma. Global Strategy for Asthma Management and Prevention; 2020. www.ginasthma.org.

13. Sousa AW, Cabral AL, Martins MA, Carvalho CR. Daily physical activity in asthmatic children with distinct severities. J Asthma. 2014;51(5):493-497.

14. Akdis CA. Does the epithelial barrier hypothesis explain the increase in allergy, autoimmunity and other chronic conditions? Nat Rev Immunol. 2021. https://doi.org/10.1038/s41577-021-00538-7. Accessed April 22, 2021 
15. BoonpiyathadT, SözenerZC,SatitsuksanoaP,Akdis CA.Immunologic mechanisms in asthma. Semin Immunol. 2019;46:101333.

16. Agache I, Akdis CA. Precision medicine and phenotypes, endotypes, genotypes, regiotypes, and theratypes of allergic diseases. J Clin Invest. 2019;129(4):1493-1503.

17. Cevhertas L, Ogulur I, Maurer DJ, et al. Advances and recent developments in asthma in 2020. Allergy. 2020;75(12):3124-3146.

18. Shi YH, Shi GC, Wan HY, et al. Coexistence of Th1/Th2 and Th17/ Treg imbalances in patients with allergic asthma. Chin Med J (Engl) . 2011;124(13):1951-1956.

19. Caminati M, Pham DL, Bagnasco D, Canonica GW. Type 2 immunity in asthma. World Allergy Organ J. 2018;11(1):13.

20. Akdis CA, Arkwright PD, Brüggen MC, et al. Type 2 immunity in the skin and lungs. Allergy. 2020;75(7):1582-1605.

21. Diamant Z, Vijverberg S, Alving K, et al. Toward clinically applicable biomarkers for asthma: an EAACl position paper. Allergy. 2019;74(10):1835-1851.

22. Walsh NP, Gleeson M, Shephard RJ, et al. Position statement. Part one: immune function and exercise. Exerc Immunol Rev. 2011;17:6-63.

23. Nieman DC, Wentz LM. The compelling link between physical activity and the body's defense system. J Sport Health Sci. 2019;8(3):201-217.

24. Del Giacco SR, Carlsen KH, Du Toit G. Allergy and sports in children. Pediatr Allergy Immunol. 2012;23(1):11-20.

25. Lowder T, Dugger K, Deshane J, Estell K, Schwiebert LM. Repeated bouts of aerobic exercise enhance regulatory $T$ cell responses in a murine asthma model. Brain Behav Immun. 2010;24(1):153-159.

26. Xepapadaki P, Bachert C, Finotto S, et al. Contribution of repeated infections in asthma persistence from preschool to school age: design and characteristics of the PreDicta cohort. Pediatr Allergy Immunol. 2018;29(4):383-393.

27. Megremis S, Niespodziana K, Cabauatan C, et al. Rhinovirus speciesspecific antibodies differentially reflect clinical outcomes in health and asthma. Am J Respir Crit Care Med. 2018;198(12):1490-1499.

28. Jartti T, Liimatainen $U$, Xepapadaki P, et al. Clinical correlates of rhinovirus infection in preschool asthma. Allergy. 2021;76(1):247-254.

29. Xepapadaki P, Korovessi P, Bachert C, et al. Evolution of airway inflammation in preschoolers with asthma-results of a two-year longitudinal study. J Clin Med. 2020;9(1):187.

30. Krug J, Kiefer A, Koelle J, et al. TLR 7/8 regulates Type I and Type III interferon signalling in RV1b induced allergic asthma. Eur Respir J. 2021;57(5):2001562.

31. Alexopoulou L, Holt AC, Medzhitov R, Flavell RA. Recognition of double-stranded RNA and activation of NF-kappaB by Toll-like receptor 3. Nature. 2001;413(6857):732-738.

32. Goodridge HS, Simmons RM, Underhill DM. Dectin-1 stimulation by Candida albicans yeast or zymosan triggers NFAT activation in macrophages and dendritic cells. J Immunol. 2007;178(5):3107-3115.

33. Firrincieli $V$, Keller A, Ehrensberger R, et al. Decreased physical activity among Head Start children with a history of wheezing: use of an accelerometer to measure activity. Pediatr Pulmonol. 2005;40(1):57-63.

34. Rabe KF, Vermeire PA, Soriano JB, Maier WC. Clinical management of asthma in 1999: the Asthma Insights and Reality in Europe (AIRE) study. Eur Respir J. 2000;16(5):802-807.

35. van Gent R, van der Ent CK, van Essen-Zandvliet LE, et al. No differences in physical activity in (un)diagnosed asthma and healthy controls. Pediatr Pulmonol. 2007;42(11):1018-1023.

36. Ownby DR, Peterson EL, Nelson D, Joseph CC, Williams LK, Johnson CC. The relationship of physical activity and percentage of body fat to the risk of asthma in 8- to 10-year-old children. J Asthma. 2007;44(10):885-889.

37. Morton AR, Fitch KD. Australian association for exercise and sports science position statement on exercise and asthma. J Sci Med Sport. 2011;14(4):312-316.
38. GINA. Global Strategy for Asthma Management and Prevention. Global Initiative for Asthma. http://www.ginasthma.org/2015

39. Lucas SR, Platts-Mills TA. Paediatric asthma and obesity. Paediatr Respir Rev. 2006;7(4):233-238.

40. Bonini M, Gramiccioni C, Fioretti D, et al. Asthma, allergy and the Olympics: a 12-year survey in elite athletes. Curr Opin Allergy Clin Immunol. 2015;15(2):184-192.

41. Gleeson M, Bishop NC, Stensel DJ, Lindley MR, Mastana SS, Nimmo MA. The anti-inflammatory effects of exercise: mechanisms and implications for the prevention and treatment of disease. Nat Rev Immunol. 2011;11(9):607-615.

42. Meiler F, Zimmermann M, Blaser K, Akdis CA, Akdis M. T-cell subsets in the pathogenesis of human asthma. Curr Allergy Asthma Rep. 2006;6(2):91-96.

43. Lambrecht $\mathrm{BN}$, Hammad $\mathrm{H}$. Allergens and the airway epithelium response: gateway to allergic sensitization. J Allergy Clin Immunol. 2014;134(3):499-507.

44. Del Giacco SR, Scorcu M, Argiolas F, Firinu D, Del Giacco GS. Exercise training, lymphocyte subsets and their cytokines production: experience of an Italian professional football team and their impact on allergy. Biomed Res Int. 2014;2014:429248.

45. Gu ZW, Wang YX, Cao ZW. Neutralization of interleukin-17 suppresses allergic rhinitis symptoms by downregulating Th2 and Th17 responses and upregulating the Treg response. Oncotarget. 2017;8(14):22361-22369.

46. Pumputiene I, Emuzyte R, Siaurys A, Tamosiunas V, Valiulis A. CD4+CD25(high) Treg cells in peripheral blood during remission and exacerbation of allergic asthma in children. Acta Paediatr. 2011;100(7):1006-1010.

47. Chakir J, Shannon J, Molet S, et al. Airway remodeling-associated mediators in moderate to severe asthma: effect of steroids on TGF-beta, IL-11, IL-17, and type I and type III collagen expression. J Allergy Clin Immunol. 2003;111(6):1293-1298.

48. Vahlkvist S, Inman MD, Pedersen S. Effect of asthma treatment on fitness, daily activity and body composition in children with asthma. Allergy. 2010;65(11):1464-1471.

49. Koster ES, Wijga AH, Raaijmakers JA, et al. High agreement between parental reported inhaled corticosteroid use and pharmacy prescription data. Pharmacoepidemiol Drug Saf. 2010;19(11):1199-1203.

50. Lambrecht BN, Hammad H, Fahy JV. The cytokines of asthma. Immunity. 2019;50(4):975-991.

51. Peters U, Dixon AE, Forno E. Obesity and asthma. J Allergy Clin Immunol. 2018;141(4):1169-1179.

52. Gao YD, Ding M, Dong $X$, et al. Risk factors for severe and critically ill COVID-19 patients: a review. Allergy. 2021;76(2):428-455.

53. Zhang Q, Bastard P, Liu Z, et al. Inborn errors of type I IFN immunity in patients with life-threatening COVID-19. Science. 2020;370(6515):eabd4570.

\section{SUPPORTING INFORMATION}

Additional supporting information may be found in the online version of the article at the publisher's website.

How to cite this article: Maurer DJ, Liu C, Xepapadaki P, et al. Physical activity in asthma control and its immune modulatory effect in asthmatic preschoolers. Allergy. 2021;00:1-15. doi:10.1111/all.15105 